\title{
Rescuing the Rescuer: Fractured Dormia Basket and a Second Basket Rescue Technique
}

\author{
Iyad Khamaysi ${ }^{1}$, Eisa Hajj ${ }^{2}$
}

\begin{abstract}
Mechanical lithotripsy is one of the well-known rescue methods for impacted common bile duct (CBD) stones. A fractured Dormia basket with a captured stone is an unusual complication and poses a special management problem. In the past, surgical intervention was the standard method. Various nonsurgical techniques have been reported. If the impaction is at the level of the papilla, extending the sphincterotomy might be sufficient. Stent insertion, endoscopic mechanical lithotripsy, extracorporeal shock wave lithotripsy, endoscopic laser lithotripsy, and transhepatic choledochoscopic lithotripsy have all been reported. In this case, we used a second basket, which is readily available and less expensive, as a rescue technique.

Keywords: Endoscopic retrograde cholangiopancreatography, Fractured Dormia Basket, Rescue.

Euroasian Journal of Hepato-Gastroenterology (2020): 10.5005/jp-journals-10018-1314
\end{abstract}

A 71-year-old woman had an 8-week history of biliary-like abdominal pain and abnormal liver function tests. A cholecystectomy was performed 15 years before presentation.

Transabdominal ultrasound and CT of the abdomen showed only CBD dilatation (up to $12 \mathrm{~mm}$ ).

Endoscopic ultrasound (EUS) showed a paradiverticular papilla and dilated CBD with a 12-mm floating stone (Fig. 1A).

During endoscopic retrograde cholangiopancreatography (ERCP), sphincterotomy was done and the stone was caught by a Dormia basket (Fig. 1A), but unfortunately, while retrieving the basket, it was impacted at the level of the papilla. A rescue mechanical lithotripsy was initiated. The duodenoscope was withdrawn and the Soehendra metal sheath was advanced over the exposed Dormia basket under fluoroscopic control (Fig. 1B). However, while cranking the lithotripter, the four wires of the basket were fractured leaving the stone/basket complex impacted in the distal CBD (Fig. 1B). Due to the paradiverticular position, the sphincterotomy could not be further extended, so a 12-mm-balloon sphincteroplasty was performed and a second Dormia basket was introduced. The stone/basket complex was disengaged; the stone was retrieved successfully but the fractured basket tip went proximally into the right hepatic duct. After many attempts, the
${ }^{1}$ Department of Gastroenterology, The Ruth and Bruce Rappaport Faculty of Medicine, Technion-Israel Institute of Technology, Haifa, Israel; Department of Gastroenterology, Rambam Health Care Campus, Haifa, Israel

${ }^{2}$ Department of Gastroenterology, Rambam Health Care Campus, Haifa, Israel

Address reprint requests to: lyad Khamaysi, Department of Gastroenterology, The Ruth and Bruce Rappaport Faculty of Medicine, Technion - Israel Institute of Technology, Haifa, Israel; Department of Gastroenterology, Rambam Health Care Campus, Haifa, Israel, Phone: +972 4777 2850, e-mail: k_iyad@rambam.health.gov.il

How to cite this article: Khamaysi I, Hajj E. Rescuing the Rescuer: Fractured Dormia Basket and a Second Basket Rescue Technique. Euroasian J Hepato-Gastroenterol 2020;10(1):45-46.

Source of support: Nil

Conflict of interest: None

fractured basket was caught (Fig. 1C) and retrieved by the second basket (Fig. 1D).

Clinically, abdominal pain and the liver tests abnormalities were resolved completely.
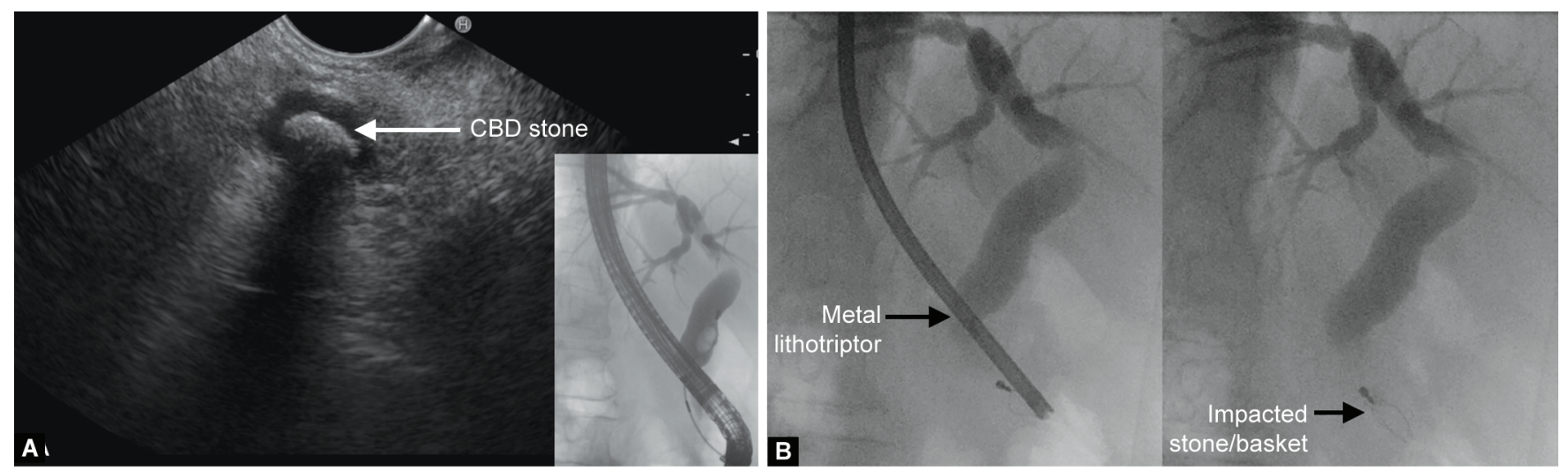

Contd...

() The Author(s). 2020 Open Access This article is distributed under the terms of the Creative Commons Attribution 4.0 International License (https://creativecommons. org/licenses/by-nc/4.0/), which permits unrestricted use, distribution, and non-commercial reproduction in any medium, provided you give appropriate credit to the original author(s) and the source, provide a link to the Creative Commons license, and indicate if changes were made. The Creative Commons Public Domain Dedication waiver (http://creativecommons.org/publicdomain/zero/1.0/) applies to the data made available in this article, unless otherwise stated. 


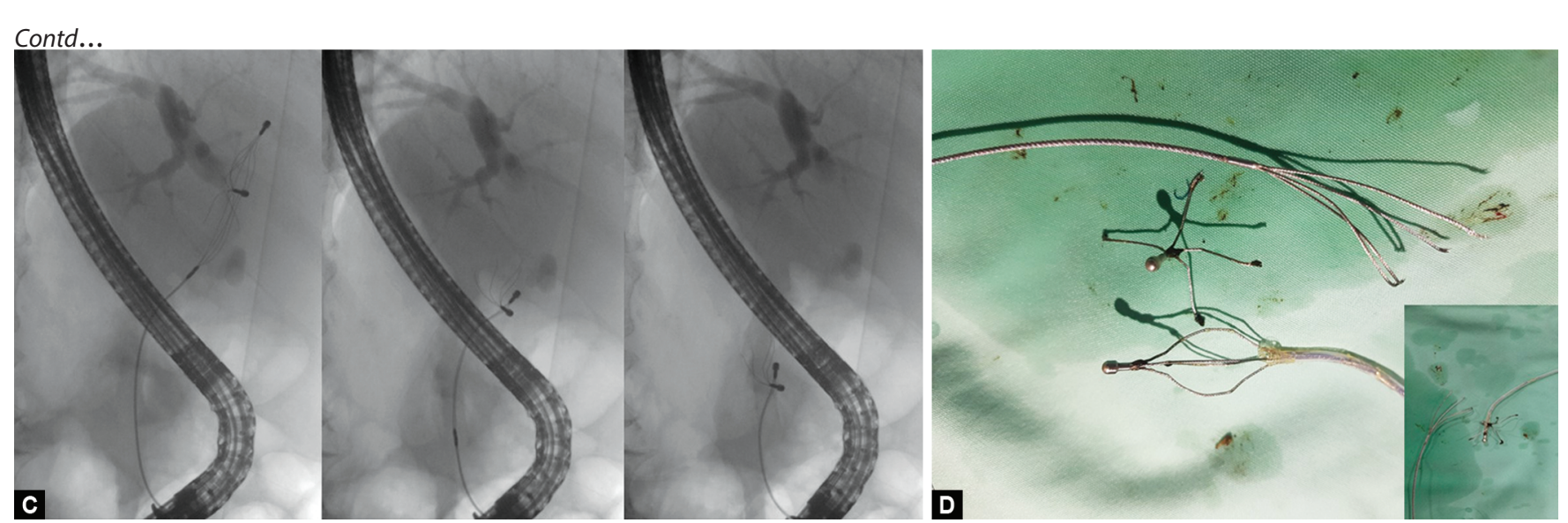

Figs 1 A to D: (A) Common bile duct (CBD) stone showed by endoscopic ultrasound and endoscopic ultrasound; (B) Stone/basket complex impacted in the distal CBD; (C) Retrieving the broken basket by a second basket; (D) Broken basket caught by a second basket

Mechanical lithotripsy is one of the well-known rescue methods for impacted CBD stones. ' A fractured Dormia basket with a captured stone is an unusual complication and poses a special management problem. In the past, surgical intervention was the standard method. Various nonsurgical techniques have been reported. If the impaction is at the level of the papilla, extending the sphincterotomy might be sufficient. Stent insertion, endoscopic mechanical lithotripsy, extracorporeal shock wave lithotripsy, endoscopic laser lithotripsy, and transhepatic choledochoscopic lithotripsy have all been reported. ${ }^{2}$

In this case, we used a second basket, which is readily available and less expensive, as a rescue technique.

To the best of our knowledge, only three similar cases, ${ }^{3,4}$ in which a second basket was used to retrieve the fractured basket and impacted stone, were published so far in English literature.

\section{References}

1. Cotton PB. Endoscopic management of bile duct stones; (apples and oranges). Gut 1984;25(6):587-597. DOI: 10.1136/gut.25.6.587.

2. Hochberger J, Tex S, Maiss J, et al. Management of difficult common bile duct stones. Gastrointest Endosc Clin N Am 2003;13(4):623-634. DOI: 10.1016/s1052-5157(03)00102-8.

3. Benatta MA, Desjeux A, Barthet $M$, et al. Impacted and fractured biliary basket: a second basket rescue technique. Case Rep Med 2016;2016:6210646. DOI: 10.1155/2016/6210646.

4. Khawaja Fl, Ahmad MM. Basketing a basket: A novel emergency rescue technique. World J Gastrointest Endosc 2012;4(9):429-431. DOI: $10.4253 /$ wjge.v4.i9.429. 